\title{
LA ESCRITURA EN UN CENTRO EDUCATIVO DE ADULTOS COMO EXPRESIÓN DE
} TRAYECTORIAS VIVIDAS

\author{
Dra. Maria del Carmen Lorenzatti (UNC) ${ }^{*}$
}

\section{RESUMEN}

En este artículo se analiza un cuento escrito por un grupo de mujeres mayores en un centro educativo de adultos de la ciudad de Córdoba (Argentina). Se realizó de manera colectiva integrando la actividad con la música y el canto. Su escritura permitió que las señoras compartan sus preocupaciones y sus opiniones sobre la importancia de la escuela en sus vidas. Desde la perspectiva de los nuevos estudios de literacidad/letramento que entiende a la cultura escrita como práctica social se observan de qué manera este cuento remite a situaciones personales de las participantes, a sus recuerdos familiares e infantiles y a sus conocimientos sobre la estructura de poder en el que se inserta la escuela. Se intenta mostrar las potencialidades de los conocimientos que las personas tienen de la lengua escrita acerca de las consecuencias de la misma. Se pretende compartir este análisis con el objeto de confrontar con las realidades de varios centros educativos visitados, donde la lengua escrita se enseña como objeto disciplinar y se produce un proceso de olvido del uso social que tiene la lengua fuera de la institución escolar. Esto tiene consecuencias negativas especialmente en los grupos de estudiantes adultos porque la escisión es grande y terminan repitiendo las letras y las sílabas de manera aislada y sin sentido. En primer lugar, se presenta el abordaje conceptual sobre cultura escrita desde los nuevos estudios de literacidad o letramento; en segundo lugar, se analiza el cuento, escrito en el marco de un proyecto de extensión universitaria con un grupo de profesionales junto a una maestra de nivel primario de jóvenes y adultos de la ciudad de Córdoba, Argentina. Finalmente, se consideran algunos puntos relacionados con la enseñanza de la lectura y escritura con jóvenes y adultos.

Palabras claves: Literacidad/letramento. Cultura escrita. Jóvenes y adultos. Trabajo. Escuelas de jóvenes y adultos.

* Docente da Facultad de Filosofia y Humanidades da Universidad nacional de Córdoba- Argentina. E-mail: marieta.lorenzatti@gmail.com 


\section{ABSTRACT}

\section{THE WRITING IN AN ADULT EDUCATION CENTER AS AN EXPRESSION OF LIVED TRAJECTORIES}

This article deals with a short story written by a group of aged women in an adult school of Córdoba (Argentina). It was written collectively, and the activity integrated also music and singing. The writing activity enabled the participating women to share their worries and their opinions about the importance of school in their lives. From the perspective of the new studies in literacy that considers the written culture as a social practice, it can be noted how this short story refers to the personal experience of the participants, their family and childhood remembrances as well as their knowledge of the power structure in which school is inserted. The intention is to show the potentiality of the knowledge people have of written language and the consequences of it. The idea is to share this analysis with the aim of bringing face to face the reality of the several schools visited, where the written language is taught as a curricular subject and where the social use of the language outside school is frequently forgotten. This provokes negative consequences specially in groups of adult students because the split is deep, and they just repeat cut off letters and syllables without making sense. First, the conceptual approach on written culture from the point of view of the new studies on literacy are explained; then the short story, written in the frame of a project of a university project with a group of professors together with a primary school teacher of young people and adults of Córdoba Argentina, is analyzed. Finally, some aspects related to the teaching of reading and writing to young people and adults are considered.

Keywords: Literacy/letramento. Written culture. Young people and adults. Work. Schools for young people and adults.

\section{RESUMO}

\section{A ESCRITA EM UM CENTRO EDUCATIVO DE ADULTOS COMO EXPRESSÃO DE TRAJETÓRIAS VIVIDAS}

Neste artigo analisa-se um conto escrito por um grupo de mulheres idosas em um centro educativo de adultos da cidade de Córdoba (Argentina). Realizouse de maneira coletiva integrando a atividade com a música e o canto. Sua escrita permitiu que as senhoras compartilhassem suas preocupações e suas opiniões sobre a importância da escola em suas vidas. A partir da perspectiva dos novos estudos de literacidade/letramento que entende a cultura escrita como prática social se observam de que maneira este conto remete a situações pessoais das participantes, suas lembranças familiares e infantis e a seus conhecimentos sobre a estrutura de poder em que se insere a escola. Tenta-se mostrar as potencialidades dos conhecimentos que as pessoas têm da língua escrita em relação às consequências da mesma. Pretende-se compartilhar esta análise com o objetivo de confrontar com as realidades de vários centros educativos visitados, onde a língua escrita é ensinada como objeto disciplinar 
e se produz um processo de esquecimento do uso social que possui a língua fora da instituição escolar. Isto tem consequências negativas especialmente nos grupos de estudantes adultos porque a cisão é grande e acabam repetindo as letras e as sílabas de maneira isoladas e sem sentido. Em primeiro lugar, se apresenta a abordagem conceitual sobre cultura escrita a partir dos novos estudos sobre literacidade ou letramento; em segundo lugar, se analisa o conto, escrito no marco de um projeto de extensão universitária com um grupo de profissionais junto a uma professora do nível primário de jovens e adultos da cidade de Córdoba, Argentina. Finalmente, se consideram alguns pontos relacionados ao ensino da leitura e escrita com jovens e adultos.

Palavras chave: Literacidade/letramento. Cultura escrita. Jovens e adultos. Trabalho. Escolas de jovens e adultos.

La discusión sobre el problema del analfabetismo tiene diversas aristas. Los organismos internacionales, los gobiernos nacionales y locales tienen preocupaciones y se ocupan de promover acciones puntuales en el intento de dar una respuesta a la problemática. Desde distintas investigaciones puedo decir que las personas que tienen baja escolaridad construyen una relación con la lengua escrita desde acciones y propósitos concretos. Sin embargo, cuando ingresan a las escuelas de adultos, la lengua escrita se presenta como objeto disciplinar y se produce un proceso de olvido del uso social que tiene la lengua fuera de la institución escolar. Esto tiene consecuencias negativas especialmente en los grupos de estudiantes adultos porque la escisión es grande y terminan repitiendo las letras y las sílabas de manera aislada y sin sentido. Frente a personas que trabajan, realizan distintos tipos de trámites administrativos, acompañan las tareas escolares de sus hijos (aún sin escribir y leer de manera convencional) las prácticas docentes en la modalidad de jóvenes y adultos muestran, en ocasiones estudiadas, que el acceso a la cultura escrita se traduce en reproducciones de fonemas y grafemas, identificación letras o sílabas sueltas y en algunos casos, la enseñanza descontextualizada de la gramática.
Estas situaciones me preocupan tanto en términos de estudio e investigación como en términos de asesoramiento específico a los maestros de jóvenes y adultos, que permanentemente se preguntan ¿cómo hacer con la lectura y escritura en el aula?.

En este artículo me interesa presentarla complejidad que significa la enseñanza en espacios educativos con personas de baja escolaridad. De manera particular analizaré un cuento que fue escrito por un grupo de mujeres mayores, de manera colectiva, en un centro educativo de adultos.

En primer lugar presentaré un breve abordaje conceptual sobre cultura escrita desde los nuevos estudios de literacidad o letramento; en segundo lugar, presento el cuento, escrito en el marco de un proyecto de extensión universitaria con un grupo de profesionales junto a una maestra de nivel primario de jóvenes y adultos de la ciudad de Córdoba, Argentina. ${ }^{1}$ Finalmente, esbozaré algunos puntos relacionados con la enseñanza de la lectura y escritura con jóvenes y adultos.

1 Se complejiza el análisis del cuento que se encuentra publicado en el libro "Saberes y conocimientos acerca de la lengua escrita. Un trabajo con maestros de jovenes y adultos (Lorenzatti, 2005). 


\section{La cultura escrita como práctica social}

Desde mediados del siglo XX, UNESCO promueve el desarrollo de planes y programas de alfabetización en distintas partes del mundo y de manera particular, en América Latina y el Caribe. En un trabajo reciente (Kalman, Lorenzatti, Mendez, Hernández, Blazich, 2018) sostengo que lo que emerge de las Conferencias Mundiales de Educación de Adultos y de los documentos de UNESCO y OEI es una visión lineal que relaciona el acceso a la cultura escrita con el progreso social; que los planes que se desarrollan plantean un trabajo acotado a la transposición del fonema al grafema; que existe una mirada que desliga la problemática de la alfabetización con otras esferas de la vida cotidiana, aunque en lo discursivo se pretenda articular los conocimientos con lo que los alumnos saben y con ello es necesario "partir de lo que los jóvenes y adultos conocen". En los últimos encuentros desarrollados por UNESCO se insiste en conceptualizar a la alfabetización como destreza y como competencia (Suwon, 2017)

De manera paralela a estas definiciones, en el mundo académico se construyen, desde la década del ' 60 , otras maneras de entender a la alfabetización o cultura escrita. En este apartado nos detendremos en la perspectiva de los nuevos estudios de literacidad (NEL o New Literacy Studies-NLS en inglés) o letramento como se lo conoce en Brasil.Zavala, et. al. (2004, p. 10) proponen el término literacidad como traducción de literacy porque entienden que "constituye una tecnología que está siempre inmersa en procesos sociales y discursivos, y que representa la práctica de lo letrado no sólo en programas escolares sino en cualquier contexto sociocultural".
Kalman y Knobel (2017) sostienenque literacidad, si bien ayuda a ampliar el concepto de alfabetización no ayuda a los lectores que no conocen el concepto de Literacy en inglés porque no aporta sobe los significados profundos que tiene este término. Las autoras hablan de prácticas del lenguaje porque incluye lo oral, lo escrito y lo multimodal. En este trabajo vamos a hablar de literacidad o cultura escrita. La literacidad no puede pensarse por fuera de lo que la gente hace con ella. No es una destreza ni competencia sino que se trata de usos con el lenguaje o prácticas discursivas particulares. En este sentido sus consecuencias serán múltiples de acuerdo con las formas en que ésta es usada por los diversos actores. (Zavala, 2005; Kalman, 2003, 2004; Baton y Hamilton, 1998)

Estos desarrollos teórico-metodológicos permiten comprender qué significa la lectura y la escritura en la vida de las personas que no tuvieron o sólo tienen un corto recorrido por la escolaridad en su infancia. Entender la cultura escrita como práctica social conduce a indagar como se entreteje la lectura y la escritura en otras prácticas. Street (1984) propone la construcción de modelos analíticos, el modelo autónomo y el modelo ideológico. El primero, analiza la literacidad como una variable independiente del contexto social, una variable autónoma cuyas consecuencias para la sociedad y para la cognición pueden derivarse de su carácter intrínseco. El modelo ideológico analiza estas prácticas como prácticas sociales por su relación con otros aspectos de vida social, ligadas no sólo a la cultura sino también a las estructuras de poder. Se incorpora a esta definición el reconocimiento de los saberes que los sujetos, en este caso, jóvenes y adultos, tienen de las consecuencias sociales de la cultura escrita. 
Kalman (2003) complejiza el concepto de prácticas letradas y analiza el conocimiento social implícito puesto en juego en las prácticas de lengua escrita desarrolladas entre los escribanos de la Plaza de Santo Domingo (México) y sus clientes. La investigadora observó en los "eventos documentales" las interacciones de los sujetos mediadas por la cultura escrita, los contextos de uso y los propósitos de quienes escribían, dejando de lado el dominio individual de habilidades y destrezas de lectura y escritura. Observó los procesos que producían ciertos tipos de textos y de qué manera emergían relaciones de poder, tanto por parte de los escribanos (cuando se negaban a considerar las ideas del cliente) como por parte de los clientes (cuando decidían qué tenía que escribir y cómo tenía que escribirse). "En este sentido el alfabetismo es doblemente un fenómeno social: los eventos de lengua escrita tienen lugar en el mundo social y, para formar parte de ellos, los participantes deben introducir en su lectura y su escritura su conocimiento de ese mundo" (Kalman, 2003, pág. 203).

Un concepto que se enmarca en esta perspectiva de los NEL es el de Multimodalidad (Kress, 2003) porque ofrece herramientas para analizar los múltiples modos de referencia que las personas usan para otorgar sentido a sus haceres. No sólo a los modos representacionales como la imagen visual, la música, los gestos, la oralidad y la escritura sino también los medios de diseminación de los mensajes. Estos modos y medios acompañan y orientan las actividades de las personas que no se apropiaron del sistema de escritura de manera convencional.

Una mirada histórica a la institución escolar permite comprender que, en Argentina, tiene un origen ligado a la enseñanza de la lengua escrita. Es su mandato fundacional y en el caso de muchas personas que sabiendo leer y escribir, aún sin haber ido a la escuela, muchas veces no se autorizan a hacerlo por no tener o no contar con la acreditación que legitima estos saberes escolares (Arrieta, 2016; Dichiara, 2017).

\section{Canto y escritura}

Quiero compartir en este apartado una experiencia de taller de escritura colectiva desarrollada en el marco de un proyecto de transferencia de investigaciones al aula (Lorenzatti, 2005). Se trató de talleres mensuales con un grupo de maestros de nivel primario de jóvenes y adultos y de otros espacios educativos no formales con el objetivo de analizar la práctica docente en relación con la lectura y escritura en grupos de baja escolaridad. Tomaré especialmente un grupo de mujeres de más de 50 años que, como producto de la escritura de un texto individual, identificaron varios momentos comunes a lo largo de su vida: nacieron y crecieron en el campo, fueron apenas uno o dos grados a la escuela, todas leen y escriben convencionalmente, en la etapa de la juventud se mudaron a vivir a la ciudad, criaron solas a sus hijos, trabajan y ahora cuidan a los nietos. Se destaca que se trata de mujeres que se conocen porque hace cuatro años que asisten al centro educativo, son amigas, y perciben a este espacio como un lugar de encuentro. Ellas sostienen que “... cualquier acontecimiento es bueno para estar juntas y si se llegan a enfermar, expresan iha!.... yo pensaba y decía ah... icómo puede ser que no pueda ir! [...] (Registro taller)

La maestra decidió abordar el tema "trabajo" y realizó un abordaje articulado con la maestra de música porque el grupo de mujeres participaba del coro. Esta integración con el género musical se produjo porque se quiso intensificar la mirada en el trabajo femenino y de esta manera, se propuso la lec- 
tura de la letra de canciones que remiten al trabajo. Se seleccionaron y cantaron dos temas que hacen referencia al trabajo: "Duerme negrito", recopilada por Atahualpa Yupanqui que trata del trabajo de una madre que realiza tareas diversas en el campo para traerle codornices, carne de cerdo y otras cosas al niño. Destaca la canción que la señora trabaja duramente y no le pagan. El segundo tema fue "Trabajo quiero Trabajo" de Yupanqui y Razzano que simboliza la ausencia de trabajo en el campo.

Ambos temas musicales despertaron comentarios de las mujeres acerca de situaciones de explotación manifestaron críticas acerca del asistencialismo del estado ya que consideran que no ayuda a cambiar la historia de las familias. También recordaron escenas de sus vidas, de sus infancias ligadas al trabajo con los animales, del trabajo de sus padres ("por el que seguramente le pagaban muy poco"), de la ausencia de escolaridad que sufrieron por tener que trabajar. Las mujeres caracterizaron a la gente de campo y mencionaron el silencio como cómplice de situaciones de explotación que vivieron sus familias y ligaron estas situaciones a la soledad vivida.

En el desarrollo de estas actividades se enfatizó la expresión oral de las estudiantes. Luego la maestra decidió solicitar la escritura de un cuento sobre la posible historia laboral de una persona imaginaria. Sugirió que escribieran tres párrafos, respetando los tres grandes momentos de la estructura narrativa: el primero presentando al personaje, lugar y tiempo de la historia. El segundo párrafo, que aborde la historia de los trabajos de ese personaje. Finalmente, pidió la historia con una valoración acerca de lo narrado. Todas escribieron larguísimos relatos autobiográficos, que tuvieron un comienzo ficcional pero pronto se convirtieron en la narración de la realidad de sus historias como trabajadoras. De todos modos, los protagonistas tuvieron otros nombres. En el momento de compartir con sus compañeras a través de la lectura de sus producciones, algunas de ellas no lo pudieron hacer pero sí tuvieron participación en la complementación desde la oralidad, cuando la maestra leía. Posteriormente las mujeres del grupo mencionaron momentos de su vida comunes en las historias contadas: infancia pobre, escuela interrumpida para irse a la ciudad a trabajar o para cuidar hermanos, maltrato de los patrones, entre otras cuestiones.

En el trabajo realizado en los talleres con los docentes se consideró importante que se pudiera promover una escritura colectiva que reuniera, desde el plano de la ficción, lo que las mujeres expresaron sobre su propia historia desde la oralidad. En este sentido, el equipo coordinador del proyecto junto a la docente del centro educativo y la maestra de música llevamos a cabo un taller con la intención de propiciar la escritura colectiva de un cuento.

Cabe señalar que ese espacio contó con la participación de todas las señoras adultas y fue dificultoso, en un primer momento, encontrar un problema que desencadene una narración. Se recuperaron las historias personales que cada estudiante había escrito y se orientó la identificación de hechos comunes en estas narraciones. Para poder superar el propio relato autobiográfico se presentó al grupo la noción de ficción, en términos de "vamos a imaginar" "vamos a hacer como si...". Y luego de un tiempo, dado que las mujeres no podían iniciar o consensuar un relato, el equipo coordinador propuso abordar un tema sentido en sus vidas. Se planteó un hipotético conflicto en torno a la continuidad de la escuela de adultos. 
A partir de esta posibilidad ficticia, cada una de ellas fue manifestando sus sentimientos en torno a la institución escolar. Las declaraciones de las mujeres evidenciaron el lugar primordial que la escuela tiene en sus vidas y se fueron entretejiendo anticipaciones de sentido en torno a lo que sería sus vidas sin la posibilidad de encontrarse en el espacio educativo.

La escritura de la narración se realizó de manera colectiva, a partir de la coordinación de la maestra y el equipo de investigadores. Se filmó la actividad, se revisaron nuevamente los videos y a partir de allí, la maestra recuperó cada momento del taller y junto a las señoras se escribió conjuntamente la narración.

A continuación, se presenta el relato escrito entre todas las estudiantes y luego se analiza su producción.

\section{El cuento colectivo: Escuela} ¡quiero una escuela! "Para

\section{ahorrar todas las amarguras que hemos tenido que aguantar".}

El título elegido por el grupo de alumnas homologa la canción de Atahualpa Yupanqui, que las mujeres cantaron en los talleres de Coro. El contexto donde se desarrollan las distintas actividades educativas se constituye en parte de la escritura colectiva. Este contexto remite a un plano multidimensional que no sólo atiende a las situaciones específicas sino también a las historias sociales e individuales que coadyuvan en la construcción de esas situaciones. (Kalman, 1998). Las mujeres apelan a lo que conocen referido al trabajo, que forma parte de sus actividades del coro, en grupo con sus compañeras. Es así que la música y la poesía que porta esa canción les permiten a las señoras imaginar una trama textual pidiendo por la continuidad de la propuesta educativa que se ofrece en ese espacio. El título de la narración colectiva se inspira en el título de la canción.

Luego de la escritura del cuento se publicó y desarrollamos un encuentro donde se le entregó una separata a cada autora. Ese momento fue muy emotivo porque cada una de ellas expresó algún decir en torno a la importancia de la escritura, de ver plasmado en un papel la producción de todas ellas y recordaron las negociaciones realizadas entre ellas y la maestra para lograr un texto coherente.

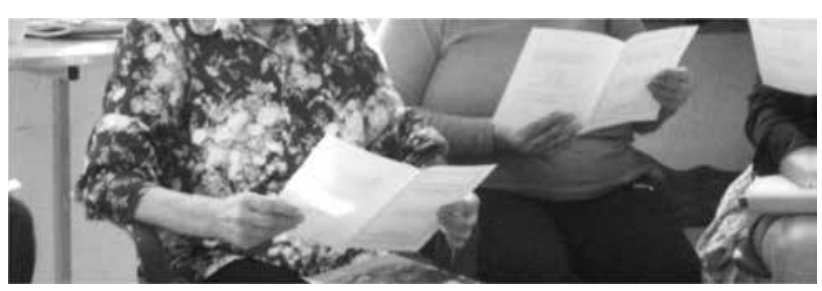

Esta foto se encuentra en la tapa del libro y muestra a dos de la mujeres en el momento en que ellas leyeron entre todas el cuento.

A continuación se transcribe el cuento.

Escuela iquiero una escuela! "Para ahorrar todas las amarguras que hemos tenido que aguantar"?

"Érase una vez un martes lluvioso del mes de noviembre, el barrio estaba tranquilo pero oscuro, como presagiando una mala noticia. La señorita Clara, maestra de adultos de ese lugar desde hacía unos años, se acerca a paso lento al portón y se sorprende al encontrar en ese horario al pastor.

- iBuenas tardes! - lo saluda.

2 Las autoras del escrito son las siguientes: $\mathrm{Au}-$ toras: Astrada Graciela, Luna Olga, Nieto Margarita, Núñez Encarnación, Cano Maria Adela , Peralta Jesús, Toranzo Ignacia , Corbalan Irma, Villarroel Tomasa, González Haidee. Luna Dora, Baigorria Mary, Contreras Teresa, Andueza Avelina, Silvina Cuello Gloria Ramos, Ester Aichino, Medina Antonia. 
- No tan buenas - dice tristemente el pastor Juan Carlos - Vengo a comunicarle que esto se cierra - y agrega apresuradamente - lo necesitamos para usarlo como guardería para los niños de madres que trabajan.

El cielo se puso más negro y Clara pensó... icómo se lo digo a mis alumnas!

En eso llega sonriendo como siempre Juanita y a medida que se acerca va transformando la sonrisa en una mueca.

- ¿Qué pasa? pregunta.

La maestra la abraza y le cuenta en pocas palabras.

- iNo lo puedo creer! - dice - ijusto ahora que vengo a aprender! Tantos años esperando... iDesde que mis padres en el campo me sacaron de $3^{\circ}$ grado para ir a trabajar! Mandé a mis hijos, después a mis nietos... y ahora que me tocaba a mí.

Poco a poco todas se fueron enterando.

- ¿Qué vamos a hacer? Ah ino! iLlamemos a la tele para que nos defienda - dice María Rosa!

- No nos vamos a ver más con el grupo. iY nos habíamos hecho tan amigas!!! El ratito que venía acá, yo me olvidaba de todos los problemas y volvía a mi casa contenta, icon ganas de seguir luchando!

- No nos demos por vencidas - dice Juanita - busquemos otro lugar, la escuela de acá a la vuelta, por ejemplo.

- Sí, pero de día no hay lugar.

- Bueno, qué importa el horario lo importante es que podamos seguir.

De golpe, como en un suspiro, se sintió la voz de Estela Maris diciendo:

- Y yo que tenía una excusa para escaparme un poquito de mi casa y pensar por fin en mí.

- A la vuelta de mi casa vive un puntero político $^{3}$ que nos puede dar una mano, qué

3 En Argentina se conoce como puntero político a se yo, puede hablar con el gobernador, con el intendente - dice María Rosa

- iMandemos una carta al Presidente! interrumpió Sonia.

- ¿Y si nos reunimos en una casa de familia? acotó María con los ojos llenos de luz como encontrando la solución definitiva.

- iHagamos una manifestación! -dijo la más revolucionaria.

Una a una fue opinando y decidieron tender hilos por todas partes.

- iUna persona sola no hace nada...pero juntas! - agregó ilusionada Jacinta.

Así comenzó una semana de idas y venidas, llamadas y súplicas, desvelos y añoranzas, trámites y más trámites pero pocas respuestas.

Finalmente, el martes siguiente las encontró reunidas, tristes y sin solución, la única diferencia era que el sol esta vez calentaba fuerte, porfiado, como exclamando iacá estoy!

.... y en eso aparece el pastor diciendo:

- iChicas! iEncontré otro lugar para la guardería! iQuédense tranquilas!!

$\mathrm{Ni}$ bombos ni tambores podrían haber hecho tanto ruido. Abrazos, risas, lágrimas y un final con mate cocido y facturitas recién hechas fueron el broche final de una semana de tanta confusión y unión".

\section{Elementos a considerar en la escritura del cuento}

En este apartado me interesa analizar, desde los nuevos estudios de literacidad, los conocimientos compartidos que van configurando el lugar de la cultura escrita en esa experiencia narrativa. El inicio de la narración toma los elementos de la realidad de las señoras, porque el centro educativo donde

las personas líderes en cuanto a poder territorial en barrios populares que tienen relaciones con autoridades de un partido político. 
ellas asisten tres días a la semana funciona en una iglesia evangélica.

La narración sigue anclada en las historias de cada una de las mujeres: la añoranza de finalizar su escolaridad primaria en la infancia, la necesidad de ir a trabajar y finalmente la decisión tomada en la adultez: “... me tocaba a mí”. Se explicita en este párrafo las trayectorias de vida, en la palabra de Juanita, de muchas mujeres que se acercan a los centros educativos: primero crían a sus hijos, viven la escolaridad a través de ellos, luego realizan lo mismo con sus nietos.

Emerge la escuela como un lugar de encuentro, de construcción de lazos de amistad. En diferentes investigaciones (Kalman, 2011; Arrieta, 2017; Andini y Paolasso, 2012) se observa que el centro educativo o escuela se constituye en un espacio para encontrarse con vecinas y compañeras con quienes comparten sus alegrías, sus tristezas, sus temores y sus logros.

En el cuento ingresa el contexto de vida de las señoras porque se evidencia el apego a las actividades escolares. En su vida cotidiana muchas veces la lectura y escritura no forma parte sistemática de sus acciones. $Y$ esperan el día de clases para realizar estas prácticas junto a sus compañeras.

Las mujeres muestran conocimientos para derivar demandas y realizar solicitudes, en este caso para defender la escuela y ver la posibilidad de encontrar otro espacio para la guardería de niños. Estas manifestaciones muestran relaciones de poder en un doble sentido: por un lado, en torno a las decisiones que se toman sobre la clausura de la escuela, y por otro lado, ellas proponen participar desde la escritura de una carta como un instrumento que posiciona a todo el grupo frente a las autoridades. La carta legitima por escrito una demanda concreta y las señoras conocen el valor de esta acción. Por eso enuncian la presencia de autoridades de diferentes instancias, tales como el presidente, el gobernador y el intendente porque ellas saben que son ellos a quien tienen que reclamar por la efectivización del derecho a la educación.

La escritura de una carta es una estrategia que se utiliza en varias campañas de alfabetización como el elemento central que acredita la finalización de la etapa alfabetizadora. En este caso, la carta es un texto que les permitirá a las mujeres expresar sus sentimientos en torno a la escuela como espacio importante en su historia de vida. La carta que se pretende escribir explicará los motivos por los cuales las mujeres defienden la escuela y pedirán su permanencia. Este texto, como modo de expresión, muestra un grupo de mujeres que solicita al estado, en el nombre de los gobernantes, que cumplan con la ley y propicien la finalización de la escolaridad de quienes no pudieron hacerlo en la edad correspondiente. También saben que la movilización puede tener resultados positivos. $\mathrm{O}$ al menos, lo intentan, lo esperan.

Este texto ficcional escrito por las mujeres muestra que la lectura y escritura son prácticas sociales situadas (Street, 1984). No sólo son actividades que se realizan sino lo que se piensan acerca de ellas, lo que se conoce acerca de las consecuencias que esa escritura tiene.

Se incorpora también en la narrativa el conocimiento que las señoras tienen acerca de la incidencia de los medios de comunicación. Según Kress (2003) estos son los medios de diseminación, aquellos que la cultura hace disponibles para distribuir los significados como mensajes (el libro, la pantalla de la computadora, la revista, el video, la película, la radio, la charla,). En este caso se apela a la "tele" (televisor) y más precisa- 
mente, los noticieros locales, identificando el lugar de los periodistas yla posibilidad de realizar una denuncia social sobre el cierre de la escuela de jóvenes y adultos donde las señoras concurren. El concepto de multimodalidad permite identificar los modos y los medios a través de los cuales las personas de baja o nula escolaridad se apropian de conocimientos y participan en la vida social. Y también ofrece herramientas para derribar ciertas apreciaciones que, desde distintos sectores, se manifiestan en torno a los sujetos que no leen y escriben de manera convencional como que son los que no saben, no opinan, no pueden realizar abstracciones por su escaso nivel de escolaridad.

El cuento narrado por las señoras muestra la posición de mujeres luchadoras, que conocen y pelean por sus derechos. Son personas letradas, pueden argumentar, hipotetizar y recurrir a otras personas que tienen otros saberes y que son pertinentes para sus demandas puntuales de continuidad de la escuela.

\section{Reflexiones finales}

A lo largo del artículo intenté poner de relieve el lugar de la cultura escrita en un centro educativo jóvenes y adultos. Lejos de recurrir a la separación en sílabas y en letras, cosa habitual en los espacios educativos de alfabetización, en este caso la lectura y escritura se relaciona con la realidad de las señoras. Se intenta reflejar una situación conflictiva que remite a un deseo de las mujeres: queremos la escuela. Y esta situación desata una serie de comentarios, que pertenecen a una ficción pero que podría ser realidad para ellas. La historia de las estadísticas que muestran que la educación de personas jóvenes y adultos son presas de las políticas de ajuste. Entonces frente a esa situación se ponen en juego las percepciones y valoraciones que las mujeres tienen del espacio escolar. La escritura es el vehículo que permite difundir la situación, objetivar las posibilidades de acción de las señoras y se constituye en la lente que objetiva sus apreciaciones.

La escritura del cuento muestra las dimensiones sociales de la cultura escrita (Kalman, 2003); es decir, muestra cómo intervienen distintos actores: la maestra, el pastor, las señoras. Todos están preocupados y ocupados por solucionar el conflicto, todos dicen algo al respecto, opinan, se manifiestan e intentan buscar soluciones. El cierre del cuento tiene un final feliz, las señoras continuarán asistiendo a la escuela y la guardería funcionará en otro lugar.

Mi experiencia en trabajos con maestros de jóvenes y adultos evidencia que son muy pocos los textos que circulan en estos espacios. Se encuentran textos cortos generalmente fotocopiados y en varias ocasiones se trata de textos escolares de niños; muchas veces son textos expositivos e instructivos, como recetas de cocina; recortes de periódicos, comics pero existe escasa producción de los estudiantes y menos aún escritura colectiva.

Por eso es necesario que en los espacios educativos se trabaje con una variedad de textos y representativos de la cultura escrita de nuestro tiempo, para que los estudiantes puedan interactuar con ellos.

Entender que la cultura escrita es una práctica social demanda a los maestros-alfabetizadores que tengan en cuenta qué saben los jóvenes y adultos de la cultura escrita, cuáles son los usos que ellos hacen con ella (Barton y Hamilton, 1998), en qué momentos de su vida cotidiana hacen uso de la lengua escrita, cómo la lengua escrita forman parte de prácticas globales más amplias.

Para finalizar, quisiera enfatizar que la apropiación de la cultura escrita tiene un 
origen social. En el caso particular de los jóvenes y adultos de nula o baja de escolaridad sabemos que arrastran historias de desvalorización porque no han ido o no han finalizado la escolaridad obligatoria. Aún en los casos de acceder a planes a distancia se les hace difícil la apropiación de la cultura escrita, porque estos programas están pensados para personas con algún trayecto de escolaridad.

Por esta razón se hace imprescindible reconocer sus conocimientos sobre la cultura escrita, validar los modos de escritura que cada uno puede tener, generar espacios y momentos de interacción con distintas personas atravesados por diferentes tipos de textos, orales, escritos, gráficos.

Es necesario trabajar de manera gradual generando situaciones que les permitan aumentar la confianza en sí mismos frente a la lectura y escritura en un trabajo colectivo y con pares. El dolor que representa no haber ido a la escuela en la edad correspondiente, aun cuando las personas, como el caso de las señoras que escribieron el cuento, sepan leer y escribir es intenso. Y se manifiesta a partir del temor a expresarse, a leer en voz alta, a compartir escrituras, entre otras maneras.

De este modo se podrá recuperar el sentido de la cultura escrita como práctica social y se caminará hacia la superación de la dicotomía alfabetizado-analfabeto que sólo aumenta el estigma hacia aquellos que no pudieron, por cuestiones sociales, acceder a la lectura y escritura de modo convencional.

\section{Referencias}

ANDINI, S. Y PAOLASSO, Y. ¿YO, SÍ PUEDOleer y escribir? Una aproximación a espacios de alfabetización de Jóvenes y Adultos. Tesis (Licenciatura en Ciencias de la Educación) Escuela de Ciencias de la Educación. Facultad de Filosofía y Humanidades. Universidad Nacional de Córdoba. 2010
ARRIETA, R. Entre la escuela y la familia: relaciones en torno al conocimiento escolar en la educación de jóvenes y adultos. En publicación con acceso on-line https://educacion-economia-trabajo-peet.org/2016jornadasjovenesyadultos/-En Jornadas: "Educación y trabajo de jóvenes y adultos a lo largo de la vida. Investigaciones y estudios acerca de las políticas, los sujetos y las experiencias en la educación de jóvenes y adultos".Facultad de Filosofía y LetrasUBA Recuperado de https://educacion-economia-trabajo-peet.org/2016jornadasjovenesyadultos/-2016

BARTON D. Y HAMILTON, M. Local literacies: Reading and writting in one community. London y New York, Routledge. , 1998

Declaración de Suwon-Osansobre la revisión a medio término de la CONFINTEA VI. El poder del aprendizajey la educaciónde adultos:una visión hacia el 2030. Instituto de de la UNESCO para el Aprendizaje a lo Largo de Toda la Vida (UIL), Hamburgo. 2017

DICHIARA, B. "Estudiar: una trama compleja de jóvenes y adultos", Tesis (Licenciatura en Ciencias de la Educación). Escuela de Ciencias de la Educación. Facultad de Filosofía y $\mathrm{Hu}$ manidades. Universidad Nacional de Córdoba. 2017

KALMAN J., LORENZATTI M. DEL C., MENDEZ A.M., HERNÁNDEZ G. BLAZICH, G.) $L a$ relevancia de la alfabetización de personas jóvenes y adultas en América Latina hoy. Centro Regional de educación de adultos de América Latina y el Caribe (CREFAL). Pátzcuaro. México. 2018

KALMAN, J. Dile que haga la comida él. En Lorenzatti, M. del C. Procesos de literacidad y educación básica de jóvenes y adultos. Narvaja editor. Córdoba 2011

Escribir en la plaza. México: Fondo de Cultura Económica. 2003

Saber lo que es la letra. Una experiencia de la lectoescritura con las mujeres de Mixquic. México: Siglo XXI. 2004

KNOBEL M. Y KALMAN J. Aprendizaje docente y nuevas prácticas de lenguaje. Posibilidades de formación en el giro digital. Biblioteca Innovación Educativa. SM de Ediciones. México.2017 
KRESS, G.Literacy in the new media age. London: Routledge.2003

LORENZATTI, M. DEL C. "Saberes y conocimientos sobre cultura escrita: un trabajo con maestros de jóvenes y adultos". Ferreyra Editor. Córdoba. 2005

LORENZATTI, M. DEL C., (2018). Conocimientosprácticas sociales y usos escolares de cultura escrita de adultos de baja escolaridad. Pátzcuaro, México: CREFAL

STREET, B., Literacy and theory practice. Cambridge: Cambridge University Press.1984

ZAVALA, V., MURCIA, M., AMES P. (Ed) Escri- tura y sociedad. Nuevas perspectivas teóricas y etnográficas (p 437-459) Lima, Perú: Red para el Desarrollo de las Ciencias Sociales en el Perú. 2004

ZAVALA, V. Un Perú que LEE, ¿un país que cambia? una mirada a los mitos de la lectoescritura, (Vol. 6). 2005

http://www.foroeducativo.org.pe/comunicaciones/revista6/zavala.htm

Recebido em: 19/11/2018

Aprovado em: 18/12/2018 